\title{
The impact of preoperative chronic kidney disease on outcomes after Crawford extent II thoracoabdominal aortic aneurysm repairs
}

\author{
Joseph S. Coselli, MD, ${ }^{\mathrm{a}, \mathrm{b}, \mathrm{c}, \mathrm{d}}$ Hiruni S. Amarasekara, MS, ${ }^{\mathrm{a}, \mathrm{b}, \mathrm{e}}$ Qianzi Zhang, MPH, \\ Ourania Preventza, MD, ${ }^{\mathrm{a}, \mathrm{b}, \mathrm{c}, \mathrm{d}}$ Kim I. de la Cruz, MD, ${ }^{\mathrm{a}, \mathrm{b}, \mathrm{c}, \mathrm{d}}$ Subhasis Chatterjee, MD, ${ }^{\mathrm{b}, \mathrm{c}, \mathrm{d}}$ \\ Matt D. Price, MS, RHIA, ${ }^{\mathrm{a}, \mathrm{b}, \mathrm{c}, \mathrm{e}}$ Susan Y. Green, MPH, ${ }^{\mathrm{a}, \mathrm{b}, \mathrm{c}, \mathrm{e}}$ and Scott A. LeMaire, MD ${ }^{\mathrm{a}, \mathrm{b}, \mathrm{c}, \mathrm{d}, \mathrm{e}}$
}

\section{ABSTRACT}

Objective: To determine whether preoperative chronic kidney disease (CKD) is predictive of poor outcomes in patients who undergo Crawford extent II thoracoabdominal aortic aneurysm (TAAA) repair.

Methods: Data were collected from patients with CKD (defined as a preoperative estimated glomerular filtration rate $<60 \mathrm{~mL} / \mathrm{min} / 1.73 \mathrm{~m}^{2} ; \mathrm{n}=399$ ) and without CKD ( $\mathrm{n}=604$ ) who underwent extent II TAAA repair during 1991 to 2016. We used univariate, multivariable, and propensity score matching analyses to compare outcomes between these 2 groups.

Results: Compared with patients without CKD, patients who presented with CKD were older and had greater rates of comorbidities, including coronary artery disease, cerebrovascular disease, and peripheral vascular disease. Patients with CKD had higher rates of operative mortality and adverse events. After propensity analysis, patients with CKD had greater rates of adverse event and renal failure necessitating dialysis, but had comparable rates of operative death to patients without CKD. Multivariable modeling indicated that $\mathrm{CKD}$ independently predicted adverse event (relative risk ratio $[\mathrm{RRR}]=1.61 ; P=.01$ ) and renal failure $(\mathrm{RRR}=1.86 ; P=.02)$ after repair. After adjustment for median age, patients with CKD had substantially worse mid-term survival than those without $(23.9 \pm 2.4 \%$ vs $48.5 \pm 2.5 \%$ at 10 years; $P<.001)$.

Conclusions: In patients who present with CKD, extent II open TAAA repair carries considerable risks of operative death and adverse events. Further investigation is needed to improve renal protection during such repair. ( $\mathrm{J}$ Thorac Cardiovasc Surg 2018;156:2053-64)

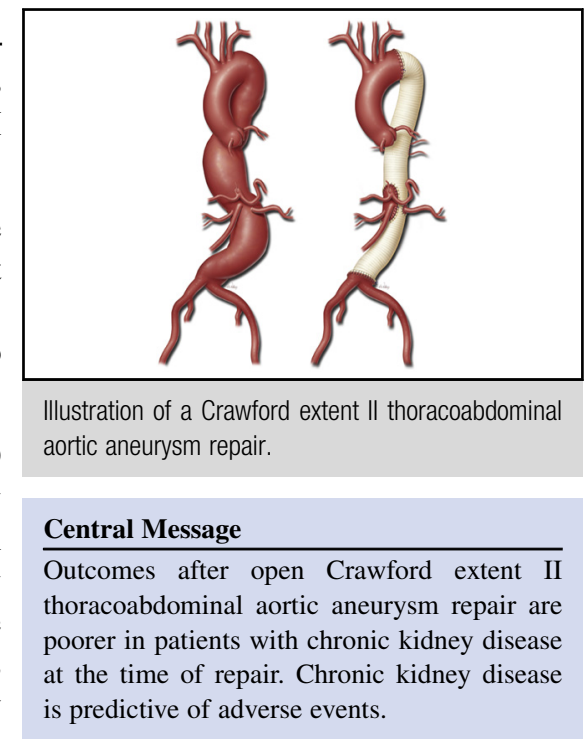

\section{Perspective}

We present the results of 1003 Crawford extent II open thoracoabdominal aortic aneurysm repairs, 399 of which were performed in patients with chronic kidney disease (CKD). Patients with CKD had higher incidences of operative death and postoperative adverse events; however, after propensity analysis, they did not have a higher mortality rate. Analysis confirmed that CKD independently predicted adverse events.

See Editorial Commentary page 2065.

See Editorial page 2049.
During the past several decades, the incidence of chronic kidney disease (CKD) has been increasing, and this increase is at least partly associated with the aging of contemporary

\footnotetext{
From the a Division of Cardiothoracic Surgery, and ${ }^{\mathrm{d}}$ Cardiovascular Research Institute, Baylor College of Medicine, Houston, Tex; ${ }^{b}$ Department of Cardiovascular

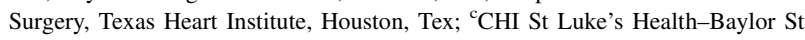
Luke's Medical Center, Houston, Tex; and 'Surgical Research Core, Michael E. DeBakey Department of Surgery, Baylor College of Medicine, Houston, Tex. Read at the 97th Annual Meeting of The American Association for Thoracic Surgery, Boston, Massachusetts, April 29-May 3, 2017.
}

populations. ${ }^{1,2}$ Cardiovascular disease is a well-established complication of $\mathrm{CKD}^{3,4}$; specifically, patients with $\mathrm{CKD}$ are predisposed to pervasive and accelerated

Received for publication May 3, 2017; revisions received May 2, 2018; accepted for publication May 28, 2018; available ahead of print July 18, 2018.

Address for reprints: Scott A. LeMaire, MD, One Baylor Plaza, BCM 390, Houston, TX 77030 (E-mail: slemaire@bcm.edu).

$0022-5223 / \$ 36.00$

Copyright (c) 2018 by The American Association for Thoracic Surgery

https://doi.org/10.1016/j.jtcvs.2018.05.101 


\section{Abbreviations and Acronyms \\ $\mathrm{CKD}=$ chronic kidney disease \\ COPD $=$ chronic obstructive pulmonary disease \\ $\mathrm{CRP}=$ cold renal perfusion \\ eGFR $=$ estimated glomerular filtration rate \\ LHB $=$ left heart bypass \\ RRR = relative risk ratio \\ TAAA $=$ thoracoabdominal aortic aneurysm}

after protocol approval, clinical data were collected prospectively, and informed consent was obtained whenever possible. A waiver of consent was approved for patients who were unable to provide consent because of illness and who had no family members available who could provide consent for them. For patients who underwent surgery before the protocol was approved, the waiver of consent was approved, and data were collected retrospectively from medical records.

Between February 1991 and August 2016, 1115 consecutive extent II open TAAA repairs were performed on our single-practice service. We were unable to obtain consent from 2 patients $(0.2 \%)$ who underwent TAAA repair after our protocol was approved; these patients were excluded from our analyses. Another 95 patients were excluded because data on preoperative estimated glomerular filtration rate (eGFR) were not available in the medical record, and 15 were excluded owing to a history of renal failure necessitating dialysis. The remaining 1003 TAAA repairs form the basis of this report.

\section{Study Definitions and Follow-up}

All data were collected using standard definitions, as reported recently. ${ }^{6,16}$ The Chronic Kidney Disease Epidemiology Collaboration equation was used to calculate eGFR on the basis of preoperative serum creatinine level, age at repair, sex, and ethnicity. ${ }^{17}$ In accordance with National Kidney Foundation Kidney Disease Outcome Quality Initiative guidelines, ${ }^{2} \mathrm{CKD}$ was defined as a preoperative eGFR $<60 \mathrm{~mL} / \mathrm{min} / 1.73 \mathrm{~m}^{2}$. According to this definition, 399 of the 1003 patients (39.8\%) had preoperative CKD, and $604(60.2 \%)$ did not. Postoperative acute renal dysfunction was defined as a peak postoperative serum creatinine level at least double the preoperative level or the need for dialysis.

Patients were considered symptomatic if they had symptoms associated with thoracoabdominal aortic disease, such as pain, hoarseness, or dysphagia. Repairs were divided into 3 eras based on the standardization of key surgical adjuncts: era 1, from 1991 to 1998, before adjuncts were standard practice; era 2, from 1999 to 2004, early adoption; and era 3, from 2005 to 2016, representing standardized contemporary use (Table E1). ${ }^{6,10}$ Operative death was defined as death that occurred within 30 days of surgery or before final discharge from our hospital or any other hospital or long-term acute care facility to which a patient might have been transferred. Adverse event was defined as a composite endpoint comprising operative death or persistent (ie, present at hospital discharge) stroke, paraplegia, paraparesis, or renal failure necessitating dialysis. ${ }^{18}$ Postoperative surveillance information was obtained through clinic visit, telephone interview, or written correspondence. The Social Security Death Index and internet obituary searches were used to identify deaths among patients who were lost to follow-up $(\mathrm{n}=25)$.

\section{Surgical Techniques}

Our overall operative strategy has been largely standardized since 2005; our surgical approach and 30-year clinical practice are described more fully elsewhere. ${ }^{6,16}$ Crawford extent II TAAA repair, the most extensive form of TAAA repair, typically involves replacing the aorta from just distal to the left subclavian artery to the aortic bifurcation and may extend into 1 or both iliac arteries. Extent II repair commonly necessitates the use of more protective adjuncts than less extensive TAAA repairs do. We routinely use moderate systemic heparinization $(1.0 \mathrm{mg} / \mathrm{kg})$, mild passive hypothermia $\left(32^{\circ} \mathrm{C}\right.$ to $\left.34^{\circ} \mathrm{C}\right)$, LHB, cerebrospinal fluid drainage, selective reattachment of intercostal or lumbar arteries, and cold renal perfusion $\left(\mathrm{CRP} ; 4^{\circ} \mathrm{C}\right)$. We selectively perfuse the celiac axis and superior mesenteric artery with isothermic blood by modifying the LHB circuit. ${ }^{10}$ In CRP, cold $\left(4^{\circ} \mathrm{C}\right)$ Ringer's lactate solution with mannitol $(12.5 \mathrm{~g} / \mathrm{L})$ and methylprednisolone $(125 \mathrm{mg} / \mathrm{L})$ is delivered through $9-\mathrm{Fr}$ Pruitt catheters as an initial bolus of 400 to $600 \mathrm{~mL}$ and then approximately every 6 to 10 minutes at a rate of 200 to $300 \mathrm{~mL} / \mathrm{min}$ for 1 or 2 minutes (Figure 1). Body temperature is carefully monitored to avoid overcooling, with CRP delivery reduced as needed. If the rectal temperature is below

\section{METHODS \\ Study Enrollment and Patient Characteristics}

Baylor College of Medicine's Institutional Review Board approved our clinical research protocol in 2006. For patients who underwent operation 


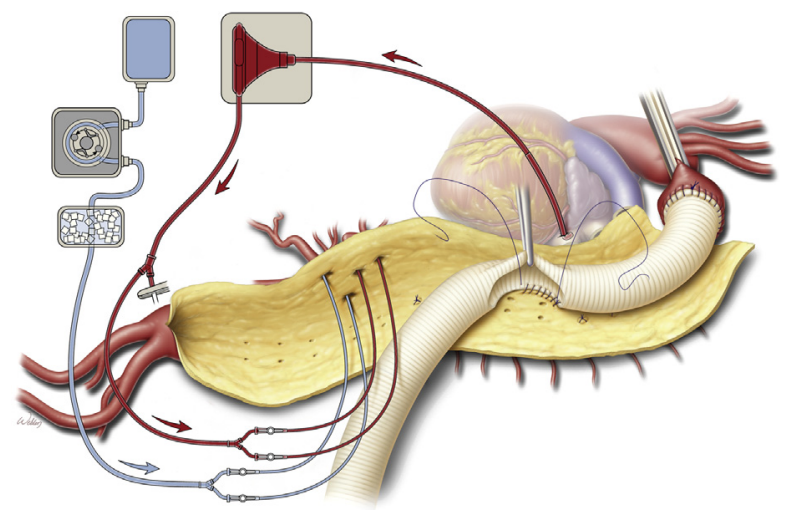

FIGURE 1. Drawing of cold renal perfusion (CRP) during Crawford extent II thoracoabdominal aortic aneurysm repair. After the proximal anastomosis is completed, the left heart bypass perfusion circuit is typically discontinued; the renal arteries are then cannulated with balloon perfusion catheters to enable intermittent perfusion with cold crystalloid solution through a separate CRP circuit. (Used with permission of Baylor College of Medicine.)

$32^{\circ} \mathrm{C}$, instead of administering CRP every 6 minutes, we increase the interval to every 12 to 18 minutes. If the rectal temperature is $31^{\circ} \mathrm{C}$ or the renal artery temperature is $15^{\circ} \mathrm{C}$, we do not administer CRP. Although CRP has been provided to most patients since 2001, certain situations may preclude its use, such as some cases of rupture and emergent repair in which the renal circuit cannot be prepared before repair begins, hemodynamic instability, some cases of dissection extending into a renal artery, and other scenarios in which inserting a balloon catheter could be dangerous.

As appropriate, we manage the visceral and renal arteries with bypass grafts, endarterectomy, and stenting. In cases of chronic aortic dissection, residual dissection is managed distally by fenestration to clear away the dissecting membrane. ${ }^{19}$ A multibranched TAAA graft may be used in cases of widely displaced visceral arteries or in patients with connective tissue disorders. In our early experience, we used the femoral artery as the arterial cannulation site during LHB; however, by 1996, we shifted to using the distal aorta as our standard site for cannulation whenever possible. In our practice, hypothermic circulatory arrest is rarely used and is reserved for aneurysms that cannot be safely clamped; likewise, a "clamp-and-sew" approach to extent II repair is rarely used, being reserved for aneurysms in which the patient's anatomy or clinical circumstances (eg, hemodynamic instability, rupture) preclude the use of LHB.

\section{Statistical Analysis}

Statistical analyses were performed with SAS version 9.4 (SAS Institute, Cary, NC), Stata version 13 (StataCorp, College Station, Tex), and $\mathrm{R}$ version 3.4.0 (R Project for Statistical Computing, Vienna, Austria). Continuous variables are presented as mean \pm standard deviation or median [interquartile range (IQR)], as appropriate. Categorical variables are presented as number and percentage. Univariate comparisons were performed using the Pearson $\chi^{2}$ test, Fisher exact test, or nonparametric Wilcoxon rank- sum test, as appropriate.

To identify independent predictors of operative death, adverse events, persistent paraplegia or paraparesis, and persistent renal failure necessitating dialysis after 1003 TAAA repairs, we built multivariable logistic regression models using clinically relevant preoperative and intraoperative factors that showed a univariate association with $P<.1$ for the event of interest (Table E2). As part of a subanalysis of the 399 repairs in the patients with CKD, we also built models to identify predictors of operative death, adverse events, and persistent renal failure necessitating dialysis (Table E3). We excluded a few variables that otherwise met the criteria for model entry because they were highly intercorrelated and then used a backward selection method with a removal $P$ value of 0.05 A variance inflation factor $\geq 10$ and clinical representability were considered. Model fit was assessed by the Hosmer-Lemeshow goodnessof-fit test and receiver operating characteristic curves. Postoperative complications were not entered into the models. Postoperative survival rates in those with and without CKD were estimated by the Kaplan-Meier method and compared by using log-rank test or Cox proportional hazard regression model adjusted for age. The assumption of proportional hazards was assessed by using the interaction term among predictors.

In addition, we analyzed outcomes using propensity-matched data for 311 pairs of patients with and without CKD. To conduct the propensity score matching, we matched observations within a \pm 0.2 caliper range. Balance of covariates in the matched data was assessed by using standardized differences $(<.10)$ and propensity score histograms with kernel density curves. The validity of the propensity scores was confirmed using the Hosmer-Lemeshow test. We assessed the similarities between the profiles (eg, means or proportions between the 2 groups) and found that the patient profiles between the groups were within $10 \%$ of the standardized mean difference (Table 1). The following preoperative variables were included in propensity score matching: age, male sex, genetic disorder, chronic dissection, diabetes, coronary artery disease, cerebrovascular disease, history of chronic obstructive pulmonary disease (COPD)/ emphysema/bronchitis, current tobacco use, acute symptoms, peripheral vascular disease, rupture, previous open distal aortic repair, and era.

\section{RESULTS}

\section{Preoperative Characteristics}

Compared with the patients without CKD, those with CKD were significantly older and had higher rates of additional comorbidities, including peripheral vascular disease $(31.1 \%$ vs $18.7 \% ; P<.001)$, coronary artery disease $(34.1 \%$ vs $24.8 \% ; P=.001)$, cerebrovascular disease $(21.6 \%$ vs $13.7 \% ; P=.001)$, and history of COPD, emphysema, or bronchitis $(40.6 \%$ vs $24.0 \%$; $P<.001$ ) (Table 1). In contrast, the patients without CKD had a higher prevalence of aortic dissection $(61.6 \%$ vs $33.3 \% ; P<.001$ ), including more frequent DeBakey type I dissection, type III dissection, and chronic dissection. In addition, the patients without CKD had higher incidences of genetically triggered aortic disease $(26.5 \%$ vs $4.0 \%)$, including Marfan syndrome $(23.0 \%$ vs $3.8 \% ; P<.001)$.

\section{Operative Details}

Patients with CKD had more overall visceral and renal procedures (ie, endarterectomy, stenting, or bypass) than those without CKD $(54.6 \%$ vs $45.4 \%, P=.004)$. In particular, endarterectomy was required in more than one-third of patients with CKD $(36.0 \%$ vs $18.9 \% ; P<.001)$. Clamping proximal to the left subclavian artery $(27.5 \%$ vs $16.5 \%$; $P<.001)$ and use of elephant trunk repair $(10.1 \%$ vs $5.3 \% ; P=.006$ ) were more common in the non-CKD group. Intercostal/lumbar artery reattachment rates were higher in the non-CKD group $(91.2 \%$ vs $77.9 \% ; P<.001)$, suggesting that there were fewer suitable arteries for 
TABLE 1. Preoperative characteristics of patients with and without CKD, overall, and propensity matched

\begin{tabular}{|c|c|c|c|c|c|c|c|c|}
\hline \multirow[b]{2}{*}{ Characteristic } & \multicolumn{4}{|c|}{ Overall } & \multicolumn{4}{|c|}{ Propensity matched cohort } \\
\hline & $\begin{array}{c}\text { All } \\
(\mathbf{n}=\mathbf{1 0 0 3})\end{array}$ & $\begin{array}{c}\text { With } \\
\text { CKD } \\
(\mathbf{n}=399) *\end{array}$ & $\begin{array}{c}\text { Without } \\
\text { CKD } \\
(n=604)\end{array}$ & $\begin{array}{c}P \\
\text { value }\end{array}$ & $\begin{array}{c}\text { All } \\
\text { matched } \\
(n=622)\end{array}$ & $\begin{array}{c}\text { With } \\
\text { CKD } \\
(\mathbf{n}=\mathbf{3 1 1})^{*}\end{array}$ & $\begin{array}{c}\text { Without } \\
\text { CKD } \\
(\mathbf{n}=311)\end{array}$ & $\begin{array}{c}\text { Standard } \\
\text { mean } \\
\text { difference }\end{array}$ \\
\hline Age, y & $65[55-72]$ & 70 [64-74] & $61[48-68]$ & $<.001$ & $68[62-72]$ & 68 [62-72] & $67[63-72]$ & .006 \\
\hline Male sex & $658(65.6)$ & $246(61.7)$ & $412(68.2)$ & .04 & $412(66.2)$ & $206(66.2)$ & $206(66.2)$ & .00 \\
\hline Genetic disorder & $176(17.5)$ & $16(4.0)$ & $160(26.5)$ & $<.001$ & $34(5.5)$ & $16(5.1)$ & $18(5.8)$ & .02 \\
\hline Marfan syndrome & $154(15.4)$ & $15(3.8)$ & $139(23.0)$ & $<.001$ & $32(5.1)$ & $15(4.8)$ & $17(5.5)$ & - \\
\hline Aortic aneurysm without dissection & 498 (49.7) & $266(66.7)$ & $232(38.4)$ & $<.001$ & $369(59.3)$ & $191(61.4)$ & $178(57.2)$ & - \\
\hline Aortic dissection & $505(50.3)$ & $133(33.3)$ & $372(61.6)$ & $<.001$ & $253(40.7)$ & $120(38.6)$ & $133(42.8)$ & - \\
\hline Acute or subacute & $52(5.2)$ & $16(4.0)$ & $36(6.0)$ & .20 & $28(4.5)$ & $11(3.6)$ & $17(5.5)$ & - \\
\hline Chronic & $453(45.2)$ & $117(29.3)$ & $336(55.6)$ & $<.001$ & $225(36.2)$ & $109(35.0)$ & $116(37.3)$ & .05 \\
\hline DeBakey type I & $212(21.1)$ & $36(9.0)$ & $176(29.1)$ & $<.001$ & $87(14.0)$ & $33(10.6)$ & $54(17.4)$ & - \\
\hline DeBakey type III & $284(28.3)$ & $91(22.8)$ & $193(32.0)$ & .002 & $158(25.4)$ & $82(26.4)$ & $76(24.4)$ & - \\
\hline DeBakey type IIIa & $32(3.2)$ & $13(3.3)$ & $19(3.2)$ & .90 & $20(3.2)$ & $10(3.2)$ & $10(3.2)$ & - \\
\hline DeBakey type IIIb & $252(25.1)$ & $78(19.5)$ & $174(28.8)$ & .001 & $138(22.2)$ & $72(23.2)$ & $66(21.2)$ & - \\
\hline Localized dissection & $9(0.9)$ & $6(1.5)$ & $3(0.5)$ & .20 & $8(1.3)$ & $5(1.6)$ & $3(1.0)$ & - \\
\hline $\begin{array}{l}\text { Maximum distal aortic diameter, } \\
\mathrm{cm}\end{array}$ & $6.3[5.7-7.3]$ & $6.4[5.9-7.3]$ & $6.3[5.6-7.3]$ & .20 & $6.4[5.8-7.2]$ & $6.3[5.8-7.2]$ & $6.5[5.7-7.4]$ & - \\
\hline Hypertension & $866(86.3)$ & $368(92.2)$ & $498(82.5)$ & $<.001$ & $557(89.5)$ & $286(92.0)$ & $271(87.1)$ & - \\
\hline Hyperlipidemia & $264(26.3)$ & $116(29.1)$ & $148(24.5)$ & .10 & $180(28.9)$ & $91(29.3)$ & 89 (28.6) & - \\
\hline Diabetes & $64(6.4)$ & $34(8.5)$ & $30(5.0)$ & .02 & $41(6.6)$ & $24(7.7)$ & $17(5.5)$ & .09 \\
\hline Coronary artery disease & $286(28.5)$ & $136(34.1)$ & $150(24.8)$ & .001 & $204(32.8)$ & $108(34.7)$ & $96(30.9)$ & .09 \\
\hline Previous CABG & 129 (12.9) & $69(17.3)$ & $60(9.9)$ & .001 & $99(15.9)$ & $58(18.6)$ & $41(13.2)$ & - \\
\hline Previous MI & $166(16.6)$ & $83(20.8)$ & 83 (13.7) & .003 & $117(18.8)$ & $66(21.2)$ & $51(16.4)$ & - \\
\hline Cerebrovascular disease & $169(16.8)$ & $86(21.6)$ & $83(13.7)$ & .001 & $111(17.8)$ & $55(17.7)$ & $56(18.0)$ & .008 \\
\hline Previous stroke & $110(11.0)$ & $51(12.8)$ & $59(9.8)$ & .10 & $74(11.9)$ & $34(10.9)$ & $40(12.9)$ & - \\
\hline $\begin{array}{l}\text { Preoperative serum creatinine level, } \\
\text { mg/dL }\end{array}$ & $1.1[0.9-1.3]$ & $1.4[1.2-1.7]$ & $0.9[0.8-1.1]$ & $<.001$ & $1.2[0.9-1.4]$ & $1.4[1.3-1.7]$ & $0.9[0.8-1.1]$ & $<.001$ \\
\hline $\mathrm{eGFR}, \mathrm{mL} / \mathrm{min} / 1.73 \mathrm{~m}^{2}$ & $67[50-87]$ & $46[37-54]$ & 83 [70-96] & $<.001$ & $60[47-75]$ & $47[37-54]$ & $75[67-87]$ & - \\
\hline Body mass index, $\mathrm{kg} / \mathrm{m}^{2}$ & $\begin{array}{l}26[23-29] \\
(\mathrm{n}=911)\end{array}$ & $\begin{array}{l}26[23-29] \\
(\mathrm{n}=359)\end{array}$ & $\begin{array}{l}26[23-29] \\
(\mathrm{n}=552)\end{array}$ & .60 & $26[23-29]$ & $26[23-30]$ & 26 [23-29] & - \\
\hline $\begin{array}{l}\text { History of COPD/emphysema/ } \\
\text { bronchitis }\end{array}$ & $307(30.6)$ & $162(40.6)$ & $145(24.0)$ & $<.001$ & $220(35.4)$ & $113(36.3)$ & $107(34.4)$ & .04 \\
\hline Current tobacco use & $781(77.9)$ & $349(87.5)$ & $432(71.5)$ & $<.001$ & $535(86.0)$ & $265(85.2)$ & $270(86.8)$ & .04 \\
\hline Symptomatic & $622(62.0)$ & $242(60.7)$ & $380(62.9)$ & .50 & $381(61.3)$ & $185(59.5)$ & $196(63.0)$ & - \\
\hline Acute & $133(13.3)$ & $51(12.8)$ & $82(13.6)$ & .70 & $76(12.2)$ & $38(12.2)$ & $38(12.2)$ & 0 \\
\hline Chronic & $520(51.8)$ & $201(50.4)$ & $319(52.8)$ & .50 & $324(52.1)$ & $155(49.8)$ & $169(54.3)$ & - \\
\hline Both acute and chronic & $31(3.1)$ & $10(2.5)$ & $21(3.5)$ & .40 & $19(3.1)$ & $8(2.6)$ & $11(3.5)$ & - \\
\hline Peripheral vascular disease & 237 (23.6) & $124(31.1)$ & $113(18.7)$ & $<.001$ & $166(26.7)$ & $85(27.3)$ & $81(26.0)$ & .03 \\
\hline Rupture & $28(2.8)$ & $13(3.3)$ & $15(2.5)$ & .50 & $16(2.6)$ & $7(2.3)$ & $9(2.9)$ & .04 \\
\hline $\begin{array}{l}\text { Previous open distal aortic } \\
\quad \text { repair } \dagger\end{array}$ & $175(17.4)$ & $85(21.3)$ & $90(14.9)$ & .009 & $129(20.7)$ & 65 (20.9) & $64(20.6)$ & .008 \\
\hline Era & & & & .005 & & & & .06 \\
\hline Era 1 & $289(28.8)$ & $115(28.8)$ & $174(28.8)$ & & $191(30.7)$ & $86(27.7)$ & $105(33.8)$ & \\
\hline Era 2 & $329(32.8)$ & $152(38.1)$ & $177(29.3)$ & & $204(32.8)$ & $114(36.7)$ & $90(28.9)$ & \\
\hline Era 3 & $385(38.4)$ & $132(33.1)$ & $253(41.9)$ & & $227(36.5)$ & $111(35.7)$ & $116(37.3)$ & \\
\hline
\end{tabular}

Values are reported as n (\%) or median [interquartile range]. $C K D$, Chronic kidney disease; $I Q R$, interquartile range; $C A B G$, coronary artery bypass graft; $M I$, myocardial infarction; eGFR, estimated glomerular filtration rate; COPD, chronic obstructive pulmonary disease. *Preoperative eGFR $<60 \mathrm{~mL} / \mathrm{min} / 1.73 \mathrm{~m}{ }^{2}$. $\nmid$ Previous open thoracoabdominal or abdominal aortic aneurysm repair. 
TABLE 2. Operative details of 1003 extent II TAAA repairs stratified by CKD

\begin{tabular}{|c|c|c|c|c|}
\hline Variable & All $(n=1003)$ & With CKD $(\mathbf{n}=399)^{*}$ & Without CKD $(n=604)$ & $P$ value \\
\hline \multicolumn{5}{|l|}{ Urgency of operation } \\
\hline Elective & $836(83.3)$ & $337(84.5)$ & $499(82.6)$ & .40 \\
\hline Urgent & $104(10.4)$ & $36(9.0)$ & $68(11.3)$ & .30 \\
\hline Emergent & $63(6.3)$ & $26(6.5)$ & $37(6.1)$ & .80 \\
\hline Reverse elephant trunk & $46(4.6)$ & $12(3.0)$ & $34(5.6)$ & .052 \\
\hline Elephant trunk completion repair & $82(8.2)$ & $21(5.3)$ & $61(10.1)$ & .006 \\
\hline Clamping proximal to left subclavian artery & $232(23.1)$ & $66(16.5)$ & $166(27.5)$ & $<.001$ \\
\hline Intercostal/lumbar artery reattachment & $862(85.9)$ & $311(77.9)$ & $551(91.2)$ & $<.001$ \\
\hline Aortic clamp time, min & $67[55-82]$ & $61[52-77]$ & 70 [59-85] & $<.001$ \\
\hline \multicolumn{5}{|l|}{ Management of visceral or renal arteries } \\
\hline Bypass graft & $338(33.7)$ & $127(31.8)$ & $211(34.9)$ & .30 \\
\hline Use of a 4-branch graft & $74(7.4)$ & $21(5.3)$ & $53(8.8)$ & .04 \\
\hline Endarterectomy & $258(25.7)$ & $144(36.0)$ & $114(18.9)$ & $<.001$ \\
\hline Stenting & $86(8.6)$ & $52(13.0)$ & $34(5.6)$ & $<.001$ \\
\hline Endarterectomy, stenting, or bypass & $492(49.1)$ & $218(54.6)$ & $274(45.4)$ & .004 \\
\hline \multicolumn{5}{|l|}{ Adjuncts } \\
\hline "Clamp-and-sew" repair & $150(15.0)$ & $71(17.8)$ & $79(13.1)$ & .047 \\
\hline Hypothermic circulatory arrest & $16(1.6)$ & $4(1.0)$ & $12(2.0)$ & .20 \\
\hline Left heart bypass & $837(83.4)$ & $324(81.2)$ & $513(84.9)$ & .10 \\
\hline \multirow[t]{2}{*}{ Left heart bypass time, $\min$} & 23 [18-29] & $21[16-27]$ & $24[20-30]$ & $<.001$ \\
\hline & $(\mathrm{n}=830)$ & $(\mathrm{n}=320)$ & $(\mathrm{n}=510)$ & \\
\hline Cannulation site: distal aorta & $762(76.0)$ & $298(74.7)$ & $464(76.8)$ & .50 \\
\hline Cannulation site: femoral artery & $65(6.5)$ & $21(5.3)$ & $44(7.3)$ & .20 \\
\hline Cerebrospinal fluid drainage & $651(64.9)$ & $255(63.9)$ & $396(65.6)$ & .60 \\
\hline Cold renal perfusion & $690(68.8)$ & $267(66.9)$ & $423(70.0)$ & .30 \\
\hline Selective perfusion of visceral arteries & $649(64.7)$ & $270(67.7)$ & $379(62.7)$ & .10 \\
\hline
\end{tabular}

Values are reported as $\mathrm{n}(\%)$ or median [interquartile range]. $C K D$, Chronic kidney disease; IQR, interquartile range. *Preoperative estimated glomerular filtration rate $<60 \mathrm{~mL} / \mathrm{min} / 1.73 \mathrm{~m}^{2}$. The distal cannulation site was unknown in 10 repairs.

reattachment in the CKD group (Table 2); this corresponded to shorter aortic clamp times in the CKD group.

\section{Early Outcomes}

There were 94 operative deaths $(9.4 \%)$ (Table 3). Although the majority $(66.0 \%)$ of operative deaths occurred $\leq 30$ days postoperatively, a substantial portion $(34.0 \%)$ occurred after 30 days. Patients with CKD had higher rates of operative mortality $(12.8 \%$ vs $7.1 \%$; $P=.003)$, adverse events $(26.3 \%$ vs $13.4 \% ; P<.001)$, persistent stroke $(5.5 \%$ vs $1.8 \% ; P<.001)$, and persistent paraplegia $(7.3 \%$ vs $3.0 \% ; P=.002)$ than those without CKD. Not surprisingly, the patients with CKD more often developed renal failure after the repair $(14.8 \%$ vs $6.8 \%$ $P<.001)$. Patients with CKD also had substantially greater postoperative rates of cardiac and pulmonary complications $(41.4 \%$ vs $27.3 \% ; P<.001$ and $50.6 \%$ vs $36.4 \% ; P<.001$, respectively). Patients with CKD were far less likely than patients without CKD to be discharged to home after repair $(59.6 \%$ vs $79.3 \% ; P<.001)$.

When patients with CKD were stratified by receipt of CRP, we did not find that CRP prevented persistent renal failure; the rates of persistent renal failure were $10.5 \%$ for patients who received CRP and $14.4 \%$ for those who did not (28/267 vs $19 / 132 ; P=.30)$. Similarly, when patients without CKD were stratified by receipt of CRP, we did not find that CRP prevented persistent renal failure; the rates of persistent renal failure were $5.2 \%$ in patients who received CRP compared with $3.9 \%$ in those who did not $(22 / 423$ vs $7 / 181 ; P=.50)$. In addition, we reviewed the use of visceral artery management approaches in patients with CKD $(\mathrm{n}=399)$ and found no difference between patients who underwent visceral artery manipulation and those who did not in terms of the development of persistent renal failure (14.9\% [27/218] vs $11.0 \%$ [20/181]; $P=.80$ ).

After propensity score matching, patients with CKD had higher rates of adverse events $(27 \%$ vs $18.6 \% ; P=.01)$, renal failure necessitating dialysis $(14.1 \%$ vs $8.7 \%$; $P=.03)$, cardiac complications $(42.8 \%$ vs $33.4 \%$; $P=.009)$, and life-altering complications $(18.1 \%$ vs $9.6 \% ; P=.005)$. Patients with CKD also had significantly longer stays in the intensive care unit (median, 6 days [IQR, 4-11 days] vs 4 days [IQR, 3-8 days]; $P=.009$ ).

Multivariable modeling indicated that CKD independently predicted adverse events (relative risk ratio [RRR], 
TABLE 3. Early outcomes

\begin{tabular}{|c|c|c|c|c|c|c|c|c|}
\hline \multirow[b]{2}{*}{ Variable } & \multicolumn{4}{|c|}{ Total cohort $(n=1003)$} & \multicolumn{4}{|c|}{ Propensity-matched cohort $(n=622)$} \\
\hline & $\begin{array}{c}\text { All } \\
(\mathrm{n}=\mathbf{1 0 0 3})\end{array}$ & $\begin{array}{c}\text { With } \\
\text { CKD } \\
(\mathbf{n}=399)^{*}\end{array}$ & $\begin{array}{c}\text { Without } \\
\text { CKD } \\
(n=604)\end{array}$ & $\begin{array}{c}P \\
\text { value }\end{array}$ & $\begin{array}{c}\text { All } \\
(n=622)\end{array}$ & $\begin{array}{c}\text { With } \\
\text { CKD } \\
(\mathbf{n}=\mathbf{3 1 1})^{*}\end{array}$ & $\begin{array}{c}\text { Without } \\
\text { CKD } \\
(\mathbf{n}=\mathbf{3 1 1})\end{array}$ & $\begin{array}{c}P \\
\text { value }\end{array}$ \\
\hline Adverse event $\dagger$ & $186(18.5)$ & $105(26.3)$ & $81(13.4)$ & $<.001$ & $142(22.8)$ & $84(27.0)$ & $58(18.6)$ & .01 \\
\hline Operative death & $94(9.4)$ & $51(12.8)$ & $43(7.1)$ & .003 & $65(10.5)$ & $34(10.9)$ & $31(10.0)$ & .70 \\
\hline $30-d$ & $62(6.2)$ & $32(8.0)$ & $30(5.0)$ & .049 & $45(7.2)$ & $23(7.4)$ & $22(7.1)$ & .90 \\
\hline Stroke & $43(4.3)$ & $29(7.3)$ & $14(2.3)$ & $<.001$ & $31(5.0)$ & $20(6.4)$ & $11(3.5)$ & .10 \\
\hline Persistent stroke $\ddagger$ & $33(3.3)$ & $22(5.5)$ & $11(1.8)$ & $<.001$ & $23(3.7)$ & $14(4.5)$ & $9(2.9)$ & .30 \\
\hline Spinal cord deficit & $134(13.4)$ & $70(17.5)$ & $64(10.6)$ & .002 & $102(16.4)$ & $57(18.3)$ & 45 (14.4) & .20 \\
\hline Persistent paraplegia & $47(4.7)$ & $29(7.3)$ & $18(3.0)$ & .002 & $39(6.3)$ & $24(7.7)$ & $15(4.8)$ & .20 \\
\hline Persistent paraparesis $\ddagger$ & $30(3.0)$ & $11(2.8)$ & $19(3.2)$ & .70 & $19(3.1)$ & $8(2.6)$ & $11(3.5)$ & .50 \\
\hline Acute renal dysfunction & $173(17.2)$ & $89(22.3)$ & $84(13.9)$ & $<.001$ & $121(19.5)$ & $70(22.5)$ & $51(16.4)$ & .054 \\
\hline Renal failure necessitating dialysis & $100(10.0)$ & $59(14.8)$ & $41(6.8)$ & $<.001$ & $71(11.4)$ & $44(14.1)$ & $27(8.7)$ & .03 \\
\hline Persistent renal failure $\ddagger$ & $76(7.8)$ & $47(11.8)$ & $29(4.8)$ & $<.001$ & $52(8.4)$ & $32(10.3)$ & $20(6.4)$ & .08 \\
\hline Cardiac complications & $330(4.8)$ & $165(41.4)$ & $165(27.3)$ & $<.001$ & $237(38.1)$ & $133(42.8)$ & $104(33.4)$ & .009 \\
\hline Pulmonary complications & $422(42.1)$ & $202(50.6)$ & $220(36.4)$ & $<.001$ & $284(45.7)$ & $151(48.6)$ & $133(42.8)$ & .20 \\
\hline Respiratory failure & $304(30.3)$ & $150(37.6)$ & $154(25.5)$ & $<.001$ & $210(33.8)$ & $111(35.7)$ & $99(31.8)$ & .30 \\
\hline Necessitating tracheostomy & $123(12.3)$ & 67 (16.8) & $56(9.3)$ & $<.001$ & $84(13.5)$ & $50(16.1)$ & $34(10.9)$ & .07 \\
\hline Bleeding necessitating reoperation & $37(3.7)$ & $18(4.5)$ & $19(3.1)$ & .30 & $23(3.7)$ & $15(4.8)$ & $8(2.6)$ & .20 \\
\hline Early survivors, $\mathrm{n}$ & 909 & 348 & 561 & & 557 & 277 & 280 & \\
\hline Life-altering complications $\S$ & $92(10.1)$ & $54(15.5)$ & $38(6.8)$ & $<.001$ & $77(13.8)$ & $50(18.1)$ & $27(9.6)$ & .005 \\
\hline Length of ICU stay, d & $\begin{array}{c}4[3-8] \\
(n=882)\end{array}$ & $\begin{array}{c}6[4-10] \\
(\mathrm{n}=335)\end{array}$ & $\begin{array}{c}4[3-7] \\
(\mathrm{n}=547)\end{array}$ & $<.001$ & $\begin{array}{c}5[3-9] \\
(\mathrm{n}=528)\end{array}$ & $\begin{array}{c}6[4-11] \\
(\mathrm{n}=265)\end{array}$ & $\begin{array}{c}4[3-8] \\
(n=263)\end{array}$ & .009 \\
\hline Length of hospital stay, $d$ & $\begin{array}{l}13[9-20] \\
(\mathrm{n}=906)\end{array}$ & $\begin{array}{l}15[11-23] \\
(\mathrm{n}=345)\end{array}$ & $\begin{array}{l}12[9-18] \\
(\mathrm{n}=561)\end{array}$ & $<.001$ & $\begin{array}{l}13[10-22] \\
(\mathrm{n}=554)\end{array}$ & $\begin{array}{l}15[11-22] \\
(\mathrm{n}=274)\end{array}$ & $\begin{array}{l}12[9-21] \\
(\mathrm{n}=280)\end{array}$ & .20 \\
\hline
\end{tabular}

Values are reported as $\mathrm{n}(\%)$ or median [interquartile range] unless otherwise noted. $C K D$, Chronic kidney disease; $I C U$, intensive care unit; $I Q R$, interquartile range. *Preoperative estimated glomerular filtration rate $<60 \mathrm{~mL} / \mathrm{min} / 1.73 \mathrm{~m}^{2} . \dagger$ Defined as operative death or persistent (present at hospital discharge) stroke, paraplegia, paraparesis, or renal failure necessitating dialysis. $¥$ Present at the time of hospital discharge or early death. §Discharge with stroke, paraplegia, paraparesis, or renal failure necessitating dialysis.

$1.61 ; P=.01)$ and renal failure necessitating hemodialysis (RRR, 1.86; $P=.02$ ) after repair, but CKD did not predict operative mortality (Table 4). CRP (RRR, $0.53 ; P=.006$ ) and intercostal/lumbar artery reattachment (RRR, 0.46; $P=.003$ ) were predicted to reduce the risk of operative mortality. Rupture was highly predictive of persistent renal failure necessitating dialysis (RRR, 4.19; $P=.004$ ), persistent paraplegia or paraparesis (RRR, 4.07; $P=.003$ ), and adverse events (RRR, 3.25; $P=.004$ ). Operative mortality in the group of 267 patients with CKD who received CRP during repair was less than one-half that of the 132 patients with CKD who did not receive CRP $(9.4 \%$ vs $19.7 \% ; P=.006)$.

Of the 399 repairs in patients with CKD, multivariable modeling showed that CRP provided protection against operative death (RRR, 0.30; $P<.001$ ), as did reattachment of intercostal and lumbar arteries (Table 5). In contrast, visceral or renal artery stenting and a history of COPD, emphysema, or bronchitis increased the risk of operative death (RRR, 2.85; $P=.009$ and RRR, 2.17; $P=.02$, respectively). In the CKD group, previous stroke was the greatest predictor of adverse events (RRR, 1.94; $P=.04$ ), and rupture was a strong predictor of persistent renal failure necessitating dialysis (RRR, $4.33 ; P=.04$ ).

Multisystem organ failure was the most common cause of operative death $(n=45 ; 48 \%)$ (Table 6$)$. Although causes of death did not appear to differ substantially between the patients with CKD and those without CKD, multisystem organ failure with sepsis was more common in patients with $\operatorname{CKD}(\mathrm{n}=19$ vs $\mathrm{n}=11)$.

\section{Late Outcomes}

Clinical follow-up data were available for 883 of the 909 (97\%) early survivors (348 with CKD and 561 without CKD); the median duration of follow-up was 5.7 years (IQR, 2.5-9.8 years). There were 519 late deaths; we were unable to identify the causes of these late deaths. Patients with CKD had substantially worse mid-term survival than those without $\mathrm{CKD}(53.2 \pm 2.6 \%$ vs $72.2 \pm 2.0 \%$ at 5 years and $23.9 \pm 2.4 \%$ vs $48.5 \pm 2.5 \%$ at 10 years; $P<.001$ ) (Figure 2, A). After adjustment for median age, the patients with CKD remained at a survival disadvantage $(23.9 \pm 2.4 \%$ vs $48.5 \pm 2.5 \%$ at 10 years; $P<.001)$ (Figure 2, B). 
TABLE 4. Multivariable analysis of 1003 extent II TAAA repairs

\begin{tabular}{lcc}
\hline \multicolumn{1}{c}{ Variable } & $\begin{array}{c}\text { Relative risk } \\
(\mathbf{9 5} \% \mathbf{C I})\end{array}$ & $\begin{array}{c}\boldsymbol{P} \\
\text { value }\end{array}$ \\
\hline Operative death $(\mathrm{n}=86)$ & $2.34(1.40-3.90)$ & .001 \\
Symptomatic & $2.25(1.20-4.24)$ & .01 \\
Stenting of visceral or renal arteries & $1.11(0.69-1.78)$ & .70 \\
Chronic kidney disease & $1.05(1.02-1.07)$ & $<.001$ \\
Increasing patient age at repair, per y & $0.53(0.33-0.84)$ & .006 \\
Cold renal perfusion & $0.46(0.27-0.77)$ & .003 \\
Intercostal/lumbar artery reattachment & & \\
Adverse event (n = 186)* & $3.25(1.44-7.33)$ & .004 \\
Rupture & $1.61(1.12-2.30)$ & .01 \\
Chronic kidney disease & $1.05(1.03-1.07)$ & $<.001$ \\
Increasing patient age at repair, per y & $1.02(1.01-1.03)$ & $<.001$ \\
Increasing aortic clamp time, per min & $0.37(0.24-0.57)$ & $<.001$ \\
Intercostal/lumbar artery reattachment & & \\
Persistent paraplegia or paraparesis $(\mathrm{n}=47)$ & & .003 \\
Rupture & $4.07(1.62-10.22)$ & .04 \\
Coronary artery disease & $1.79(1.10-2.92)$ & .04 \\
Chronic kidney disease & $1.25(0.76-2.05)$ & .40 \\
Intercostal/lumbar artery reattachment & $0.50(0.28-0.87)$ & .01 \\
Genetic disorder & $0.33(0.11-0.93)$ & .04 \\
Renal failure necessitating dialysis $(\mathrm{n}=76)$ & & \\
Rupture & $4.19(1.56-11.20)$ & .004 \\
Chronic kidney disease & $1.86(1.11-3.12)$ & .02 \\
Increasing patient age at repair, per y & $1.07(1.04-1.10)$ & $<.001$ \\
Increasing aortic clamp time, per min & $1.02(1.01-1.04)$ & $<.001$ \\
\hline
\end{tabular}

$C I$, Confidence interval. *Defined as operative death or discharge with renal failure necessitating dialysis, paraplegia, paraparesis, or stroke.

\section{DISCUSSION}

In patients undergoing extent II TAAA repair, those with CKD had considerably worse outcomes than those without CKD. Furthermore, CKD was found to be an independent predictor of adverse events and renal failure necessitating dialysis. Although the identification of CKD as a predictor of these key postoperative complications is not entirely surprising, our results indicate that CKD is a useful preoperative factor to assess and should be considered when counseling patients about the potential outcomes of open TAAA repair. Importantly, CKD is relatively easy to assess, and patients with CKD tend to have substantial comorbidities commonly considered to enhance risk during TAAA repair. ${ }^{6,20}$ The well-established cardiovascular complications, as well as pervasive atherosclerosis, in patients with CKD may contribute to poor outcomes after extensive TAAA repair.

The patients with CKD in our group were far older than the other patients and appeared more likely to present for extensive TAAA repair stemming from an atherosclerotic pathway, rather than from chronic aortic dissection or a connective tissue disorder. Specifically, CKD involves the development of secondary conditions, such as renal parenchymal hypertension and dyslipidemia, which then amplify the risk of atherosclerosis. ${ }^{4}$ Thus, patients with
TABLE 5. Multivariable analysis of 399 extent II TAAA repairs with CKD

\begin{tabular}{lll}
\hline \multicolumn{1}{c}{ Variable } & $\begin{array}{c}\text { Relative risk } \\
(\mathbf{9 5} \% \mathbf{C I})\end{array}$ & $\begin{array}{c}\boldsymbol{P} \\
\text { value }\end{array}$ \\
\hline Operative death $(\mathrm{n}=51)$ & & \\
Stenting of visceral or renal arteries & $2.85(1.30-6.27)$ & .009 \\
History of COPD/emphysema/bronchitis & $2.17(1.15-4.07)$ & .02 \\
Left heart bypass & $1.18(0.52-2.70)$ & .70 \\
Intercostal/lumbar artery reattachment & $0.33(0.17-0.63)$ & .001 \\
Cold renal perfusion & $0.30(0.16-0.58)$ & $<.001$ \\
Adverse event (n=105)* & & \\
Previous stroke & $1.94(1.03-3.68)$ & .04 \\
Increasing aortic clamp time, per min & $1.02(1.01-1.03)$ & .001 \\
Left heart bypass & $0.81(0.45-1.48)$ & .50 \\
Cold renal perfusion & $0.62(0.38-1.01)$ & .06 \\
Intercostal/lumbar artery reattachment & $0.32(0.19-0.56)$ & $<.001$ \\
Persistent renal failure necessitating & & \\
$\quad$ dialysis (n $=47)$ & & \\
Rupture & $4.33(1.06-17.70)$ & .04 \\
Increasing age at repair, per y & $1.06(1.02-1.11)$ & .008 \\
Increasing aortic clamp time, per min & $1.02(1.01-1.04)$ & .002 \\
Increasing eGFR, per mL/min/1.73 $\mathrm{m}^{2}$ & $0.96(0.93-0.99)$ & .003 \\
Left heart bypass & $0.73(0.32-1.67)$ & .45 \\
Cold renal perfusion & $0.68(0.35-1.32)$ & .30 \\
\hline
\end{tabular}

CI, Confidence interval; COPD, chronic obstructive pulmonary disease $e G F R$, estimated glomerular filtration rate. *Defined as operative death or discharge with renal failure necessitating dialysis, paraplegia, paraparesis, or stroke.

CKD are more likely than those without CKD to develop cardiovascular disease and more likely to have worse outcomes. ${ }^{21}$ Although the development of cardiovascular disease is considered multifactorial in origin, there is evidence implicating CKD itself as an independent risk factor for cardiovascular disease. ${ }^{22-24}$ Furthermore, the increased atherosclerotic burden in patients with CKD can impact technical aspects of TAAA repair. For example, we reattached intercostal and lumbar arteries less

TABLE 6. Details regarding 94 operative deaths after 1003 extent II TAAA repairs

\begin{tabular}{lcc}
\hline \multicolumn{1}{c}{ Variable } & $\begin{array}{c}\text { With CKD } \\
(\mathbf{n = 5 1 )}, \mathbf{n}\end{array}$ & $\begin{array}{c}\text { Without CKD } \\
(\mathbf{n}=\mathbf{4 3}), \mathbf{n}\end{array}$ \\
\hline Time of operative death & & \\
POD $\leq 30$ & 31 & 29 \\
POD $>30$ & 20 & 14 \\
Primary system(s) contributing to death & & \\
Cardiac & 6 & 4 \\
Cerebral & 6 & 6 \\
Mesenteric & 4 & 3 \\
Pulmonary & 2 & 5 \\
Multisystem & 26 & 19 \\
With renal failure & 16 & 15 \\
With sepsis & 19 & 11 \\
With renal failure and sepsis & 12 & 9 \\
Unknown or other & 6 & 5 \\
\hline
\end{tabular}

$C K D$, Chronic kidney disease; $P O D$, postoperative day. 

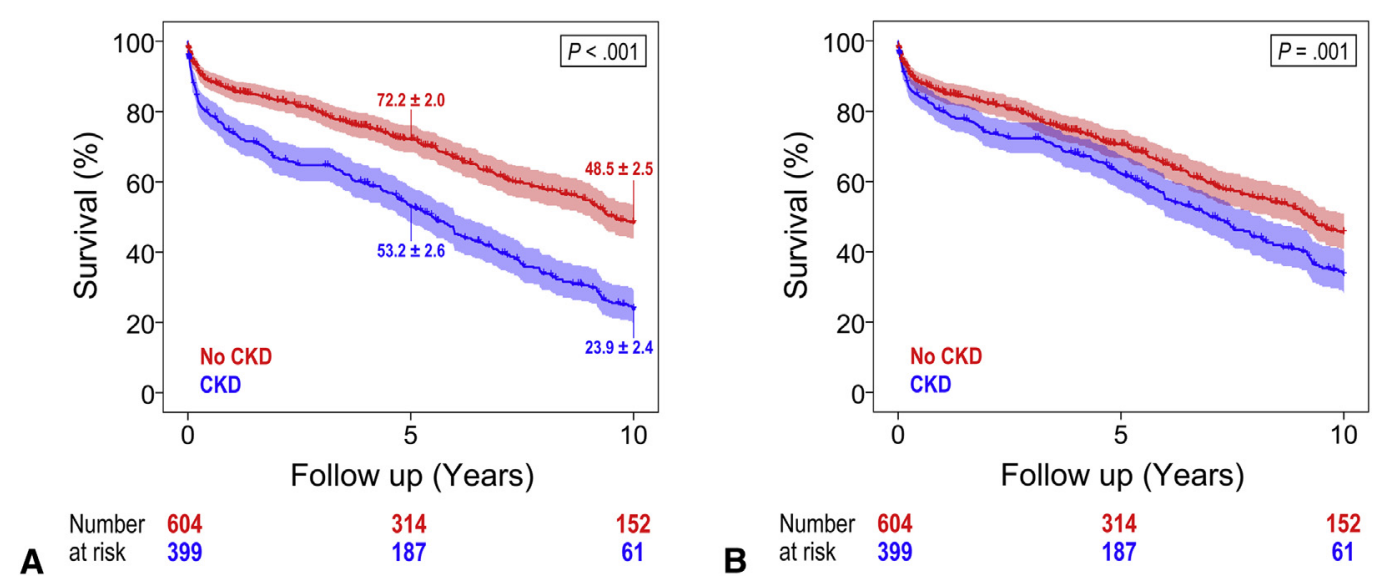

FIGURE 2. Estimated survival after 1003 extent II thoracoabdominal aortic aneurysm repairs stratified by chronic kidney disease $($ CKD) status $(n=399)$. A, Kaplan-Meier curve showing a survival disadvantage for patients with CKD $(P<.001)$. B, When we adjusted for age using a Cox regression model to analyze survival, a survival disadvantage remained for those patients with CKD (hazard ratio [HR], 1.38; 95\% confidence interval [CI], 1.14-1.66, $P=.001$ ). Each year of age also was associated with poorer survival (HR, 1.04; $95 \% \mathrm{CI}, 1.03-1.05 ; P<.001)$. Survival curves were formulated from the data of all patients and include 94 operative deaths. CIs are shown in the shaded bands.

frequently in the CKD group, perhaps because there were fewer patients with patent vessels suitable for reattachment. Similarly, as expected, branch vessel endarterectomy, stenting, and bypass were performed much more often in the patients with CKD.

Interestingly, although CKD was an independent predictor of adverse events and renal failure necessitating dialysis, it was not a predictor of operative death. Of the 100 patients who experienced postoperative renal failure, $59(59 \%)$ survived. Among the early survivors, $23(39 \%)$ were taken off dialysis before discharge. Early survivors with postoperative renal failure had higher rates of $\mathrm{CKD}$ $(61 \%$ vs $56.1 \% ; P=.60)$ and CRP $(71.2 \%$ vs $61 \%$; $P=.30)$ than patients who experienced operative death; however, neither of these findings is significant. The propensity score analysis supported the finding that patients with CKD did not have a greater rate of operative death. Survival after the development of postoperative renal failure may be associated with the increased use of CRP. In a multivariable analysis of all extent II repairs, CRP was protective against operative mortality but not against renal failure necessitating dialysis, supporting the hypothesis that CRP may offer a survival benefit for patients who develop postoperative renal failure. Conversely, CRP might have been identified as protective for operative mortality merely because of the small sample size of the operative death outcome itself; this possibility requires further investigation.

Although the impact of preoperative CKD (as defined by eGFR) on outcomes after extent II TAAA repair has not been specifically studied, the role of renal function on TAAA repair has been pursued by other investigators. $^{7,15,22,25,26}$ In 2017 report by Girardi and colleagues ${ }^{25}$ of 202 patients with preoperative renal dysfunction (defined as serum creatinine $>1.5 \mathrm{mg} / \mathrm{dL}$ or the need for dialysis) out of a total of 711 patients undergoing descending thoracic or TAAA repair, logistic regression analysis identified impaired renal function as a predictor of operative mortality; in addition, when the data were isolated for 96 extent II TAAA repairs, there was a substantial difference in operative mortality rate between the patients with renal disease and those without renal disease $(28.5 \%$ vs $4.9 \% ; P=.001)$. Predictive modeling by Safi and colleagues $^{26}$ showed that other variables prognostic of postoperative renal failure included preoperative creatinine level, providing visceral perfusion, left renal artery reattachment, and a "clamp-and-sew" approach to repair. Furthermore, in our recent report of our 30-year experience with all extents of TAAA repair, chronic renal insufficiency (serum creatinine level $\geq 3.0 \mathrm{mg} / \mathrm{dL}$ or requiring dialysis) was found to be an independent predictor of adverse events and operative mortality. ${ }^{6}$

Clearly, the role of patient-specific comorbidities, especially compromised renal function, plays a large role in the development of postoperative complications. Notably, aortic rupture was a stronger predictor than CKD in terms of predicting adverse event (RRR, 3.25 vs 1.61) and renal failure necessitating dialysis (RRR, 4.19 vs 1.86). Like CKD, rupture was not predictive of operative death, but it did predict persistent paraplegia or paraparesis (RRR, 4.07; $P=.003)$. Although we have not seen dramatic improvements in the rates of postoperative renal failure from our previous study focused on 442 extent II TAAA repairs, we believe that the use of contemporary renal protective adjuncts is beneficial. Notably, patients with CKD who received CRP were only one-half as likely to die as those who did not receive it; analysis identified CRP as independently protective against operative mortality 
(RRR, $0.30 ; P<.001)$. Rupture was highly predictive of renal failure necessitating dialysis in a subanalysis of patients with CKD (RRR, 4.33; $P=.04$ ).

Nonetheless, assessing the role of renal-specific protective adjuncts in TAAA repair is difficult, especially in patients with compromised renal function. The use of renal adjuncts tends to vary greatly between established centers: LHB, profound hypothermic circulatory arrest, the temperature of renal artery perfusion, and the solutions used for renal artery perfusion all vary. ${ }^{7-9,12,14,15,25,26}$ This was highlighted by Svensson and colleagues' retrospective appraisal of E. Stanley Crawford's attempts to find predictors of reduced acute renal failure after 1233 descending thoracic or TAAA repairs. ${ }^{7}$ In this analysis, the protection against developing acute renal failure provided by LHB, isothermic blood renal perfusion, and cold crystalloid renal perfusion were assessed; however, no protective relationship could be established-instead, preexisting renal dysfunction, along with diffuse atherosclerosis, the use of the LHB, and markers of hemodynamic instability were identified as independent predictors of acute renal failure. ${ }^{7}$ To add confusion, others have also identified renal or visceral perfusion as predictive of adverse events. ${ }^{25,26}$

Perioperative management of patients with CKD relies on collaboration between the surgeon, intensivist, and nephrologist; multidisciplinary intensive care unit rounding is ideal. Primary aims are to ensure adequate mean arterial pressure and volume resuscitation, as well as to recognize developing cardiac, pulmonary, or infectious complications. We focus attention on maintaining glycemic control in patients with diabetes, careful monitoring of potassium and other electrolytes, and avoiding premature restarting of renin-angiotensin antagonists. Thoughtful consideration for intravascular access is maintained because hemodialysis access may eventually be needed. This includes avoiding peripherally inserted central catheters and subclavian venous lines, if possible, because they pose a risk of long-term venous stenosis. ${ }^{27}$ After TAAA repair, the need for imaging before discharge is carefully balanced against the risk of contrast nephropathy. Every attempt is made to use the lowest possible volume of nonionic, iso-osmolar contrast agent. We wait until the serum creatinine level has returned as close to baseline as possible before imaging. Intravenous hydration with $1 \mathrm{~L}$ of crystalloid before the test and with another $500 \mathrm{~mL}$ afterward is routine. In patients deemed at higher risk, we add $\mathrm{N}$-acetylcysteine to further reduce the risk of contrast nephropathy. ${ }^{28}$

Limitations of our study include the fact that our data come from a single practice, and our experience might not be universal. In addition, data were collected both retrospectively and prospectively from patients treated over a nearly 3-decade period, during which renal protection strategies and data collection procedures evolved substantially. The latter factor limits our ability to analyze the effect of surgical era on postoperative outcomes (as we have shown previously). ${ }^{6,10}$ We believe that the propensity score matching analysis between the CKD and non-CKD groups is also subject to possible confounding, because patients with CKD are more likely to have preoperative atherosclerotic comorbidities, such as coronary artery disease, peripheral vascular disease, and so on. Even when matched for these preoperative atherosclerotic-based characteristics, it is difficult to tease apart the atherosclerotic origin of CKD; do the associated atherosclerotic comorbidities occur before the development of CKD, or does CKD predispose patients to the development of these comorbidities? Because this question has not been fully elucidated, the inferential power of the propensity score analysis may be limited. Moreover, we may have failed to capture all late deaths.

In conclusion, CKD as indicated by eGFR may be a useful tool in assessing individual risk among patients undergoing extensive aortic repair. Because patients with CKD tend to have many overlapping comorbidities that also tend to enhance operative risk, it may serve as a useful "primary marker" to identify patients at greatest operative risk. Patients with CKD face a substantial risk of postoperative complications after extensive TAAA repair; more than $25 \%$ of such patients will have a major adverse event after repair, and more than $15 \%$ will be discharged with a life-altering complication (eg, persistent stroke, paraparesis, paraplegia, new-onset renal failure necessitating dialysis). Preoperative counseling may help provide a realistic assessment of operative risk in patients with CKD that is balanced against the far greater risk presented by rupture in cases in which repair is inappropriately delayed. There remains a great need for new approaches to optimizing kidney protection during these extensive procedures.

\section{Webcast}

You can watch a Webcast of this AATS meeting presentation by going to: https://aats.blob.core.windows. net/media/17AM/2017-05-02/RM311/05-02-17_Room311_ 1442_Coselli.mp4.

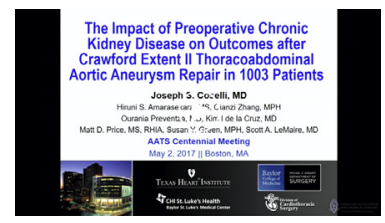

\section{Conflict of Interest Statement}

J.S.C. participates in clinical research trials conducted by Glaxo Smith Kline and Bolton Medical; consults for, 
receives royalties and a departmental educational grant from, and participates in clinical trials for Vascutek Terumo; and consults and participates in clinical trials for Medtronic and WL Gore \& Associates. O.P. consults for and participates in clinical trials for Medtronic. S.A.L. has served as a consultant for Medtronic and Vascutek Terumo, an Advisory Board Member for Baxter Healthcare, a principal investigator for clinical studies sponsored by Vascutek Terumo and Baxter Healthcare, and a coinvestigator for clinical studies sponsored by Medtronic, WL Gore \& Associates, Glaxo Smith Kline, Cook, and CytoSorbents. All other authors have nothing to disclose with regard to commercial support.

The authors thank Laurie Fondren for managing subject enrollment; Scott A. Weldon, MA, CMI, for creating the illustrations; and Stephen N. Palmer, PhD, ELS, for providing editorial support.

\section{References}

1. Coresh J, Selvin E, Stevens LA, Manzi J, Kusek JW, Eggers P, et al. Prevalence of chronic kidney disease in the United States. JAMA. 2007;298:2038-47.

2. Kidney Disease: Improving Global Outcomes (KDIGO) CKD Work Group. KDIGO 2012 Clinical practice guideline for the evaluation and management of chronic kidney disease. Kidney Int Suppl. 2013;3:1-150.

3. National Kidney Foundation. K/DOQI clinical practice guidelines for chronic kidney disease: evaluation, classification, and stratification. Am J Kidney Dis. 2002:39(2 Suppl 1):S1-266.

4. Balla S, Nusair MB, Alpert MA. Risk factors for atherosclerosis in patients with chronic kidney disease: recognition and management. Curr Opin Pharmacol. 2013;13:192-9.

5. Olechnowicz-Tietz S, Gluba A, Paradowska A, Banach M, Rysz J. The risk of atherosclerosis in patients with chronic kidney disease. Int Urol Nephrol. 2013; 45:1605-12.

6. Coselli JS, LeMaire SA, Preventza O, de la Cruz KI, Cooley DA, Price MD, et al. Outcomes of 3309 thoracoabdominal aortic aneurysm repairs. J Thorac Cardiovasc Surg. 2016;151:1323-37.

7. Svensson LG, Coselli JS, Safi HJ, Hess KR, Crawford ES. Appraisal of adjuncts to prevent acute renal failure after surgery on the thoracic or thoracoabdominal aorta. J Vasc Surg. 1989;10:230-9.

8. Aftab M, Coselli JS. Reprint of: Renal and visceral protection in thoracoabdominal aortic surgery. J Thorac Cardiovasc Surg. 2015; 149(2 Suppl):S130-3.

9. Bhamidipati CM, Coselli JS, LeMaire SA. Perfusion techniques for renal protection during thoracoabdominal aortic surgery. J Extra Corpor Technol. 2012;44:P31-7.

10. Coselli JS, de la Cruz KI, Preventza O, LeMaire SA, Weldon SA. Extent II thoracoabdominal aortic aneurysm repair: how I do it. Semin Thorac Cardiovasc Surg. 2016;28:221-37.

11. Köksoy C, LeMaire SA, Curling PE, Raskin SA, Schmittling ZC, Conklin LD, et al. Renal perfusion during thoracoabdominal aortic operations: cold crystalloid is superior to normothermic blood. Ann Thorac Surg. 2002;73:730-8

12. Kouchoukos NT, Kulik A, Castner CF. Outcomes after thoracoabdominal aortic aneurysm repair using hypothermic circulatory arrest. J Thorac Cardiovasc Surg. 2013;145(3 Suppl):S139-41.

13. LeMaire SA, Jones MM, Conklin LD, Carter SA, Criddell MD, Wang XL, et al. Randomized comparison of cold blood and cold crystalloid renal perfusion for renal protection during thoracoabdominal aortic aneurysm repair. J Vasc Surg. 2009;49:11-9; discussion 19.

14. Tshomba Y, Kahlberg A, Melissano G, Coppi G, Marone E, Ferrari D, et al Comparison of renal perfusion solutions during thoracoabdominal aortic aneurysm repair. J Vasc Surg. 2014;59:623-33.

15. Wynn MM, Acher C, Marks E, Engelbert T, Acher CW. Postoperative renal failure in thoracoabdominal aortic aneurysm repair with simple cross-clamp technique and $4^{\circ} \mathrm{C}$ renal perfusion. J Vasc Surg. 2015;61:611-22.
16. LeMaire SA, Price MD, Green SY, Zarda S, Coselli JS. Results of open thoracoabdominal aortic aneurysm repair. Ann Cardiothorac Surg. 2012;1: 286-92.

17. Levey AS, Stevens LA, Schmid CH, Zhang YL, Castro AF III, Feldman HI, et al. A new equation to estimate glomerular filtration rate. Ann Intern Med. 2009;150: 604-12.

18. LeMaire SA, Miller CC III, Conklin LD, Schmittling ZC, Köksoy C, Coselli JS A new predictive model for adverse outcomes after elective thoracoabdominal aortic aneurysm repair. Ann Thorac Surg. 2001;71:1233-8.

19. Coselli JS, LeMaire SA, Weldon SA. Extent II repair of thoracoabdominal aortic aneurysm secondary to chronic dissection. Ann Cardiothorac Surg. 2012;1: 394-7.

20. Svensson LG, Crawford ES, Hess KR, Coselli JS, Safi HJ. Experience with 1509 patients undergoing thoracoabdominal aortic operations. J Vasc Surg. 1993;17: 357-68; discussion 368-70.

21. Weiner DE, Tabatabai S, Tighiouart H, Elsayed E, Bansal N, Griffith J, et al. Cardiovascular outcomes and all-cause mortality: exploring the interaction between CKD and cardiovascular disease. Am J Kidney Dis. 2006;48:392-401.

22. Miller CC III, Villa MA, Achouh P, Estrera AL, Azizzadeh A, Coogan SM, et al. Intraoperative skeletal muscle ischemia contributes to risk of renal dysfunction following thoracoabdominal aortic repair. Eur J Cardiothorac Surg. 2008;33: 691-4.

23. Sarnak MJ, Levey AS, Schoolwerth AC, Coresh J, Culleton B, Hamm LL, et al. Kidney disease as a risk factor for development of cardiovascular disease: a statement from the American Heart Association Councils on Kidney in Cardiovascular Disease, High Blood Pressure Research, Clinical Cardiology, and Epidemiology and Prevention. Circulation. 2003;108:2154-69.

24. van der Zee S, Baber U, Elmariah S, Winston J, Fuster V. Cardiovascular risk factors in patients with chronic kidney disease. Nat Rev Cardiol. 2009;6:580-9.

25. Girardi LN, Ohmes LB, Lau C, Di Franco A, Gambardella I, Elsayed M, et al. Open repair of descending thoracic and thoracoabdominal aortic aneurysms in patients with preoperative renal failure. Eur J Cardiothorac Surg. 2017;51:971-7.

26. Safi HJ, Harlin SA, Miller CC, Iliopoulos DC, Joshi A, Mohasci TG, et al. Predictive factors for acute renal failure in thoracic and thoracoabdominal aortic aneurysm surgery. J Vasc Surg. 1996;24:338-44; discussion 344-5.

27. Hoggard J, Saad T, Schon D, Vesely TM, Royer T. Guidelines for venous access in patients with chronic kidney disease. A Position Statement from the American Society of Diagnostic and Interventional Nephrology, Clinical Practice Committee and the Association for Vascular Access. Semin Dial. 2008;21:186-91.

28. Kwok CS, Pang CL, Yeong JK, Loke YK. Measures used to treat contrast-induced nephropathy: overview of reviews. Br J Radiol. 2013;86:20120272.

Key Words: aneurysm (aorta), aortic operation, aortic dissection, thoracoabdominal, kidney disease, outcomes

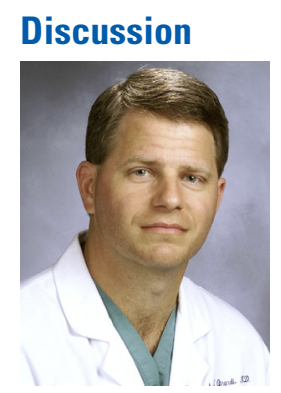

Dr L. Girardi (New York, NY). Thank you for the privilege of discussing this paper. I would like to congratulate Dr Coselli and his team yet again for sharing with us their extensive experience on thoracoabdominal aneurysm repair. I am quite certain that we will never again see a presentation with more than 1000 extent IIs unless, of course, you come up with another analysis, in which case we will see that again at some point in the future.

To summarize, patients with chronic kidney disease, not surprisingly, were older with a much greater atherosclerotic burden and with COPD, similar to others who have looked at this process as well; in multivariable analysis, chronic kidney disease patients had a much higher incidence of 
postoperative renal failure and need for hemodialysis, and a composite adverse event outcome of mortality, spinal cord injury, stroke, and dialysis. Surprisingly, mortality was not affected dramatically. Their 5- and 10-year survivals were compromised.

As you outlined, your group has spent a lot of time looking at renal protection in the past utilizing cold renal perfusion, crystalloid, and in 2 previous trials you had shown this to be a benefit as far as kidney preservation and also to be just as good as cold blood perfusion. If you look at the total cohort of patients here, 1003, in both groups, about $65 \%$ to $70 \%$ of the patients had cold renal perfusion as their primary renal protective strategy.

So given that in an extent II, for the most part, renal artery access is not an issue, what happened to the other $31 \%$ ? Was it mostly from another era, perhaps in that first era I, or was it those patients who got cold blood perfusion were not included?

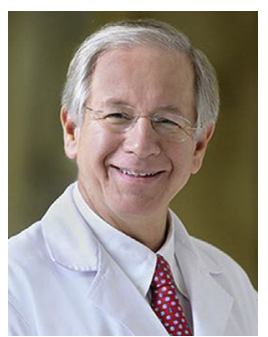

Dr J. Coselli (Houston, Tex). It's a combination of all those items, and thank you, Len, for your kind comments. In the early part of this series, we were not yet using cold crystalloid renal perfusion, and because the analysis is for the entire group, the lack of providing cold renal perfusion to nearly a third of cases is primarily related to the time this technique was adopted. Additionally, there were several years where we carried out 2 prospective randomized clinical trials, so that kept us from fully implementing this technique until these trials were completed. I believe this helps explain why cold renal perfusion of the kidneys was not used in more patients. Our contemporary use is a lot higher.

Dr Girardi. So basically if you have an extent II and even a III and a IV, for the most part you would advocate for using cold renal perfusion as often as you can?

Dr Coselli. We would and we do.

Dr Girardi. Thank you. My second question is, chronic kidney disease clearly predicted a higher incidence of postoperative renal failure on multivariable analysis; you had a relative risk of approximately 1.86. Interestingly, however, $24 \%$ of the patients who had postoperative need for dialysis subsequently recovered their renal function and left the hospital off dialysis, which is fairly gratifying.

When you look at the percentage of patients in each group, chronic kidney disease and those without kidney disease, those with it who developed postop need for dialysis had a lower rate of recovery than those without chronic kidney disease-again, not surprising. But was there a difference in your use of cold renal perfusion in those 2 recovering groups? Could you see a benefit in adding recovery, not just in the incidence of hemodialysis, but also in recovery if you used cold renal perfusion?

Dr Coselli. For the first part, we do not have the precise data on the number of patients discharged on hemodialysis who then came off hemodialysis; certainly, this has happened, but it is a limitation of our data that we just don't have concrete data on late (postdischarge) renal recovery. As you mentioned, there were 24 early survivors of extent II thoracoabdominal aortic aneurysm repair with chronic kidney disease that had postoperative renal failure, and, interestingly, 19 of those are dead now and 5 are still alive. We had 12 patients in each group (those with chronic kidney disease and those without) that recovered kidney function prior to hospital discharge; of these 24,17 received cold renal perfusion and $7 \mathrm{did}$ not (and of these 7, 6 received left heart bypass and 1 received hypothermic circulatory arrest). For those with chronic kidney disease who recovered (that is, postoperative renal dialysis was transient and did not persist to hospital discharge), 10 out of the 12 received cold perfusion and the other 2 had left heart bypass. For those without chronic kidney disease that underwent transient dialysis, 7 of 12 received cold perfusion.

However, the bottom line here is that despite the fact that we are analyzing a huge number of extent II TAAA repairs, when we get down to the specifics of renal recovery, the numbers are probably too small to make a statistically significant recommendation, but anecdotally, the trend is toward a favorable scenario when cold renal perfusion is used.

Dr Girardi. It is amazing that they recover after all that.

Dr Coselli. They do.

Dr Girardi. Patients with chronic kidney disease underwent a lot more adjunctive procedures, including stenting and endarterectomy and bypasses. In a previous study that you and Scott had done, you had shown that certainly in fragile renal arteries that underwent endarterectomy there was actually a higher incidence of postoperative dialysis if those procedures were performed in that population. Given that more than $50 \%$ of the patients in this series had those procedures, did those procedures predict better renal outcome in both groups and in the overall cohort, and then if so, do you advocate for doing that routinely if they have a lesion, or is there even a role for looking at these patients preoperatively and doing some things earlier, since $85 \%$ of them in fact are elective?

Dr Coselli. The trend is toward using these adjuvants; specifically, we advocate for renal artery stenting and endarterectomy if indicated. In the manuscript, we included the use of renal artery bypass grafts alongside renal artery stenting and endarterectomy in the renal artery reintervention variable; however, we actually use bypass grafts for different reasons. Bypass grafts are often used 
in cases of chronic dissection when the anatomic origins of the arteries become displaced, and to reattach them properly, an interposition graft is helpful, whereas endarterectomy and stenting is focused on patients with renal artery occlusive disease, and so that actually confounds our analysis somewhat. But again, we would still recommend that in patients with renal artery stenosis, this condition should be dealt with intraoperatively.

Additionally, you raise a very interesting suggestion. It may be that in order to improve postoperative renal function in patients with chronic kidney disease, you would have to exclude patients with severe renal disease in acute crisis, including those who present with ruptures or other emergencies. Perhaps we should be thinking about renal disease more like we do coronary artery disease; maybe those patients with renal artery stenosis who have recoverable renal function (not those with unrecoverable function who might have a little peanut-sized kidney) should first undergo elective intervention on the kidney before undertaking extensive thoracoabdominal aortic aneurysm repair.

Dr Girardi. Thank you.

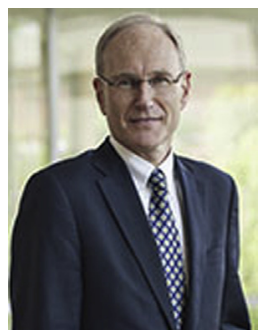

Dr S. Bolling (Ann Arbor, Mich). Was there a separate group of patients that you operated on using deep hypothermia in the thoracoabdominal group and compared with those patients with renal failure?

Dr Coselli. We do not routinely use deep hypothermic circulatory arrest (HCA) for TAAA repair. In this series, we used deep HCA for specific and uncommon circumstances, such as when we cannot get proximal control of the descending thoracic aorta to place a cross-clamp on the aorta or for other anatomic reasons. In this series of extent II TAAA repairs, the numbers of cases where we used HCA was really too small to make any kind of statistical evaluation that made clinical sense. 
TABLE E1. Era of surgical repair for 1003 extent II repairs stratified by CKD

\begin{tabular}{cccc}
\hline Variable & All $(\mathbf{n}=\mathbf{1 0 0 3})$ & With CKD $(\mathbf{n}=\mathbf{3 9 9})^{*}$ & Without CKD $(\mathbf{n}=\mathbf{6 0 4})$ \\
\hline Era of repair & & & $P$ value \\
Era 1 (1991-1998) & $289(28.8)$ & $115(28.8)$ & $174(28.8)$ \\
Era 2 (1999-2004) & $329(32.8)$ & $152(38.1)$ & $177(29.3)$ \\
Era 3 (2005-2016) & $385(38.2)$ & $132(33.1)$ & $253(41.9)$ \\
\hline
\end{tabular}

$C K D$, Chronic kidney disease. *Preoperative estimated glomerular filtration rate $<60 \mathrm{~mL} / \mathrm{min} / 1.73 \mathrm{~m}^{2}$.

TABLE E2. Variables entered into multivariable logistic regression models

\begin{tabular}{ll}
\hline \multicolumn{1}{c}{ Preoperative variables } & \multicolumn{1}{c}{ Operative variables } \\
\hline Model for operative death & \\
Age at admission & Emergent repair \\
Symptomatic & Prior open distal aortic repair \\
Chronic dissection & Visceral or renal endarterectomy \\
Genetic disorder & Visceral or renal stenting \\
Chronic kidney disease & Intercostal reattachment \\
Cerebrovascular disease & Cold renal perfusion \\
Coronary artery disease & \\
Peripheral vascular disease & \\
Pulmonary disease & \\
Rupture & \\
\hline Model for adverse event & \\
Age at admission & Emergent repair \\
Symptomatic & Visceral or renal bypass \\
Chronic dissection & Visceral or renal endarterectomy \\
DeBakey type I & Visceral or renal stenting \\
Genetic disorder & Aortic clamp time \\
Coronary artery disease & Intercostal/lumbar artery reattachment \\
Peripheral vascular disease & \\
Pulmonary disease & \\
Rupture & Intercostal/lumbar artery reattachment \\
\hline Model for persistent paraplegia & \\
Age at admission & Emergent repair \\
Symptomatic & Visceral or renal endarterectomy \\
Chronic dissection & Clamp-and-sew \\
Genetic disorder & Intercostal/lumbar artery reattachment \\
Coronary artery disease & \\
Pulmonary disease & \\
Rupture & \\
Chronic kidney disease & \\
Model for persistent renal failure necessitating dialysis \\
Age at admission & Visceral or renal bypass \\
Chronic dissection & Aortic clamp time \\
Genetic disorder & \\
Diabetes & \\
Rupture & \\
\hline
\end{tabular}

*Adverse event is defined as operative death or discharge with renal failure necessitating dialysis, paraplegia, paraparesis, or stroke.
TABLE E3. Variables entered into multivariable logistic regression models for patients with CKD $(n=399)$

\begin{tabular}{ll}
\hline \multicolumn{1}{c}{ Preoperative variables } & \multicolumn{1}{c}{ Operative variables } \\
\hline $\begin{array}{l}\text { Model for operative death } \\
\text { Symptomatic }\end{array}$ & Elective repair \\
Previous stroke & Visceral or renal endarterectomy \\
Coronary artery disease & Visceral or renal stenting \\
History of COPD/emphysema/ & Intercostal reattachment \\
bronchitis & Cold renal perfusion \\
& Left heart bypass \\
Model for adverse event* & \\
Previous stroke & Visceral or renal bypass \\
& Intercostal reattachment \\
& Cold renal perfusion \\
& Left heart bypass \\
Model for persistent renal failure & necessitating dialysis \\
Age at admission & Visceral or renal bypass \\
Rupture & Cold renal perfusion \\
eGFR & Left heart bypass \\
& Aortic clamp time \\
\hline
\end{tabular}

$C O P D$, Chronic pulmonary obstructive disease; $e G R F$, estimated glomerular filtration rate. *Adverse event is defined as operative death or discharge with renal failure necessitating dialysis, paraplegia, paraparesis, or stroke. In univariate analysis, left heart bypass did not meet criteria for entering the three models and was forced into the model. Likewise, cold renal perfusion did not meet criteria for entering either the adverse event model or the persistent renal failure necessitating dialysis mode and was forced into the model. 